\title{
Quasi-normal modes in topologically massive gravity
}

\author{
Ivo Sachs \\ Arnold Sommerfeld Center for Theoretical Physics (ASC), \\ Ludwig-Maximilians Universität, \\ Theresienstrasse 37, D-80333, München, Germany \\ E-mail: Ivo.Sachs@physik.uni-muenchen.de
}

\section{Sergey N. Solodukhin}

Laboratoire de Mathématiques et Physique Théorique CNRS-UMR 6083, Université de Tours, Parc de Grandmont, 37200 Tours, France, and

CERN Theory Division, CH 1211 Geneva 23, Switzerland

E-mail: solodukh@lmpt.univ-tours.fr

ABSTRACT: We determine the black hole quasi-normal mode spectrum for tensor perturbations in topologically massive AdS-gravity. In the special case of chiral gravity quasi-normal modes are absent despite of the presence of a horizon. In the process we uncover a simple algebraic structure in the quasi normal modes spectrum: the tower of QNM's consists of descendents of a "chiral highest weight" QNM which in turn satisfies a first order equation.

Keywords: AdS-CFT Correspondence, Black Holes in String Theory, Black Holes. 


\section{Contents}

1. Introduction 1

2. Preliminaries 2

3. Quasi-normal modes 1

3.1 Local $\mathrm{SL}(2, R) \times \mathrm{SL}(2, R)$ algebra of Killing vector fields

3.2 Scalar modes 5

3.3 Tensor modes 8

4. Conclusion 10

\section{Introduction}

The question whether and in what sense 3-dimensional gravity with a negative cosmological constant makes sense as a unitary quantum theory has a long history. Since there are no propagating bulk degrees of freedom the dynamics appears to be entirely contained in the boundary degrees of freedom. This was made precise in the re-formulation of 3dimensional gravity as a pair of Chern-Simons Lagrangians [1], 2] which was subsequently reduced to a Liouville theory on the boundary of $A d S_{3}$ [3, 迆. A neccessary condition for the viability of any quantum theory of $A d S_{3}$ gravity is the requirement that the semiclassical properties of the 3-dimensional BTZ-black holes [5] are correctly encoded in the microscopic theory. While the semi-classical Hawking radiation [6] is successfully reproduced in this formulation $[7,8$, Liouville theory fails to reproduce the entropy of the 3-dimensional BTZblack holes [9]. This inconsistency can be removed by embedding $A d S_{3}$-gravity into super string theory on $A d S_{3} \times S^{3} \times M^{4}$ 《10 which is dual to $Q_{5}$ D5 - and $Q_{1}$ D1 brane system on $M^{4} \times S^{1}$. This is a large $N$ duality in the sense that the supergravity approximation is valid for $g_{s} \rightarrow 0, N=\sqrt{Q_{1} Q_{5}} \rightarrow \infty$. Apart from enlarging the field content of $A d S_{3}$-gravity to the full supergravity multiplet, one is faced with the question of the validity of this duality at finite $N \gg 1$.

It was already noticed in [11] that the retarded 2-pt function in a BTZ black hole background decays exponentially in time whereas at finite $\mathrm{N}$ the dual CFT is Poincarérecurrent [12]. A possible resolution may be to replace the SUGRA background by a weighted sum over all asymptoically AdS geometries as in [13]. However, unless the contributions from geometries with horizon has zero measure, Poincaré recurrence cannot possibly be reproduced on the gravity side [12]. Another logical possibility is to replace the SUGRA background by a sum over micro-state geometries as suggested in [14. The BTZ horizon should then appear as a result of corse graining. 
Recently, arguments were given in [15] in favor of a duality between pure gravity on $A d S_{3}$ and a holomorphically factorized CFT. Now holomorphic factorization implies Poincaré recurrence trivially. On the other hand, asymptotically AdS geometries with a horizon contribute to the partition function of the dual CFT. Since pure Einstein gravity with a negative cosmological constant has no propagating degrees of freedom the presence of a horizon does not need to contradict holomorphic factorization. Of course, a scalar field does propagate on the black hole back ground and the corresponding quasi-normal modes imply exponential decay of the real-time correlator. But a scalar field is not a degree of freedom in pure gravity. On the other hand, Einstein gravity in 3-dimensions can be supplemented with a gravitational Chern-Simon term [16]. In that case [15] produces evidence that for integer values of the cosmological constant this theory may be dual to a pair of left/right CFT's with different central charges. The difference is that the topologically massive theory obtained in this way has a propagating graviton so that we expect generically that the presence of a horizon will spoil holomorphic factorization of the dual CFT at finite temperature.

In the present paper we analyse the structure of gravitational quasi-normal modes for topologically massive gravity. We find a infinite set of quasi-normal modes in the BTZbackgroud for generic values of the CS-coupling thus indicating the exponential decay of the retarded correlation function of the energy-momentum tensor in the "dual CFT". The case of chiral gravity is special. Although there is a BTZ-black hole in chiral topological gravity there is no gravitational quasi-normal mode associated with it. This is then consistent with the proposal that this theory is dual to chiral CFT on the boundary. For generic values of the CS-coupling, however it appears that the only way we can possibly recover Poincaré recurrence on the gravity side is by summing over a continuum of non-geometrical configurations, possibly by treating the spin-connection and the 3-bein as independent variables, in such a way that the geometric contributions with horizon have zero measure.

\section{Preliminaries}

We take as the starting point the action

$$
S=-\frac{1}{\kappa^{2}} \int\left(R+\frac{2}{l^{2}}\right)+\frac{1}{4 m \kappa^{2}} \int\left(\epsilon^{\mu \nu \alpha} R_{\mu \nu a b} \omega_{, \alpha}^{a b}+\frac{2}{3} \omega_{b, \mu}^{a} \omega_{c, \nu}^{b} \omega_{a, \alpha}^{c}\right)
$$

where $\kappa^{2}=16 \pi G$. The parameter $l$ sets the AdS scale, in what follows we will use units with $l=1$.

The parameter $m$ is related to the levels $k_{L / R}$ in the Chern-Simons formulation of 3d gravity [15] through $\mathrm{m}=\left(k_{R}+k_{L}\right) /\left(k_{R}-k_{L}\right)$. In particular, if one assumes that the quantization condition for $k$ in CS-theory applies, then $m$ is a rational number with $|m| \geq 1$. The action (2.1) leads to the equation of motion

$$
(1)_{\mu \nu}+(2)_{\mu \nu}=0
$$


where

$$
\begin{aligned}
& (1)_{\mu \nu}=R_{\mu \nu}-\frac{1}{2} g_{\mu \nu} R-g_{\mu \nu} \\
& (2)_{\mu \nu}=\frac{1}{m} \epsilon_{\mu}^{\alpha \lambda} \nabla_{\alpha}\left(R_{\lambda \nu}-\frac{1}{4} g_{\lambda \nu} R\right)
\end{aligned}
$$

Notice, that the solution to these equations is space-time with constant scalar curvature, $R=-6$. The equation for the linear metric perturbations is then given by the variation of the equation of motion. This gives

$$
\begin{aligned}
\delta(1)_{\mu \nu}= & \frac{1}{2} \nabla_{\kappa} \nabla_{\nu} h_{\mu}^{\kappa}+\frac{1}{2} \nabla_{\kappa} \nabla_{\mu} h_{\nu}^{\kappa}-\frac{1}{2} \nabla^{2} h_{\mu \nu}-\frac{1}{2} \nabla_{\mu} \nabla_{\nu} h_{\kappa}^{\kappa}+2 h_{\mu \nu} \\
& -\frac{1}{2} g_{\mu \nu} \nabla^{\kappa} \nabla^{\gamma} h_{\kappa \gamma}+\frac{1}{2} g_{\mu \nu} \nabla^{2} h_{\kappa}^{\kappa}-g_{\mu \nu} h_{\kappa}^{\kappa} \\
\delta(2)_{\mu \nu}= & \frac{1}{4 m}\left[\epsilon_{\mu}^{\alpha \lambda} \nabla_{\alpha} h_{\lambda \nu}+\epsilon_{\nu}^{\alpha \lambda} \nabla_{\alpha} h_{\lambda \mu}\right] \\
& +\frac{1}{4 m}\left[\epsilon_{\mu}^{\alpha \lambda} \nabla_{\alpha} \nabla_{\kappa} \nabla_{\nu} h_{\lambda}^{\kappa}+\epsilon_{\nu}^{\alpha \lambda} \nabla_{\alpha} \nabla_{\kappa} \nabla_{\mu} h_{\lambda}^{\kappa}\right] \\
& -\frac{1}{4 m}\left[\epsilon_{\mu}^{\alpha \lambda} \nabla_{\alpha} \nabla^{2} h_{\lambda \nu}+\epsilon_{\nu}^{\alpha \lambda} \nabla_{\alpha} \nabla^{2} h_{\lambda \mu}\right]
\end{aligned}
$$

To continue we need to fix the gauge. We impose

$$
\nabla^{\mu} h_{\mu \nu}=0, \quad g^{\mu \nu} h_{\mu \nu}=0
$$

With this choice the equations

$$
\delta(1)_{\mu \nu}+\delta(2)_{\mu \nu}=0
$$

for the linear perturbations on the background of locally AdS metric with the Riemann curvature $R_{\alpha \beta}^{\mu \nu}=-\left(\delta_{\alpha}^{\mu} \delta_{\beta}^{\nu}-\delta_{\beta}^{\mu} \delta_{\alpha}^{\nu}\right)$, take a simple form

$$
\left(\nabla^{2}+2\right) h_{\mu \nu}+\frac{1}{m} \epsilon_{\mu}^{\alpha \beta} \nabla_{\alpha}\left(\nabla^{2}+2\right) h_{\beta \nu}=0
$$

or equivalently, since the two differential operators in (2.6) commute,

$$
\left(\nabla^{2}+2\right)\left[\epsilon_{\mu}^{\alpha \beta} \nabla_{\alpha} h_{\beta \nu}+m h_{\mu \nu}\right]=0 .
$$

The equations of motion for the linear perturbations thus split on two equations: the equation for a massless graviton $\left(\nabla^{2}+2\right) h_{\mu \nu}=0$ which does not have propagating solutions and the first order equation for a massive graviton

$$
\left(\epsilon_{\mu}^{\alpha \beta} \nabla_{\alpha} h_{\beta \nu}+m\right) h_{\mu \nu}=0 .
$$

Notice that the gauge conditions (2.5) follow automatically from (2.8) which thus contains information about both the field equations and the gauge. The parameter $m$ introduced as coupling in the gravitational action (2.1) thus has the meaning of the mass of the graviton. It is interesting that the originally higher derivative equations of motion reduce to a first order in derivative equation (2.8). This fact seems to be rather general [17] and is valid for any massive field of spin $s \geq 1$ in three-fimensional AdS background. 
Squaring the equation (2.8) produces the second order equation

$$
\left(\nabla^{2}+3-m^{2}\right) h_{\mu \nu}=0
$$

In what follows we will, however, work directly with the first order equation (2.8) in order to avoid ambiguities with the sign of $m$ present in (2.9).

In the AdS/CFT correspondence the bulk theory of topologically massive gravity is equivalent to a conformal field theory with brocken local Lorentz invariance. This theory is characterized by different left and right central charges 18, 19]

$$
c_{L}=\frac{3}{2 G}\left(1+\frac{1}{m}\right), \quad c_{R}=\frac{3}{2 G}\left(1-\frac{1}{m}\right) .
$$

The values $m=1$ and $m=-1$ are special in that one of the central charges vanishes and the dual CFT contains only one chiral sector.

The change in the Riemann tensor produced by the metric perturbation (over AdS background) that satisfies equation (2.9) is

$$
\delta R_{\alpha \beta}^{\mu \nu}=\frac{\left(1-m^{2}\right)}{2}\left(h_{\alpha}^{\mu} \delta_{\beta}^{\nu}+h_{\beta}^{\nu} \delta_{\alpha}^{\mu}-h_{\beta}^{\mu} \delta_{\alpha}^{\nu}-h_{\alpha}^{\nu} \delta_{\beta}^{\mu}\right)
$$

so that the perturbation with $m^{2}=1$ produces no change in the curvature and thus, locally, is a pure gauge (see also [20-22] and [23] for a related discussion).

\section{Quasi-normal modes}

\subsection{Local $\mathrm{SL}(2, R) \times \mathrm{SL}(2, R)$ algebra of Killing vector fields}

The black hole solutions of Einstein Gravity with a negative cosmological constant are also solutions in topologically massive gravity. Here we consider the non-rotating ${ }^{1}$ black hole with unit mass given by the metric

$$
d s^{2}=-\sinh ^{2}(\rho) d \tau^{2}+\cosh ^{2}(\rho) d \phi^{2}+d \rho^{2} .
$$

In what follows we will work in light cone coordinates $u=\tau+\phi, v=\tau-\phi$, in which

$$
g=\left(\begin{array}{ccc}
\frac{1}{4} & -\frac{1}{4} \cosh (2 \rho) & 0 \\
-\frac{1}{4} \cosh (2 \rho) & \frac{1}{4} & 0 \\
0 & 0 & 1
\end{array}\right) .
$$

The black hole metric (3.2) admits two sets of the Killing vector fields, $L_{k}$ and $\bar{L}_{k}, k=$ $0,-1,1$ defined as

$$
\begin{aligned}
L_{0} & =-\partial_{u} \\
L_{-1} & =e^{-u}\left(-\frac{\cosh (2 \rho)}{\sinh (2 \rho)} \partial_{u}-\frac{1}{\sinh (2 \rho)} \partial_{v}-\frac{1}{2} \partial_{\rho}\right) \\
L_{1} & =e^{u}\left(-\frac{\cosh (2 \rho)}{\sinh (2 \rho)} \partial_{u}-\frac{1}{\sinh (2 \rho)} \partial_{v}+\frac{1}{2} \partial_{\rho}\right)
\end{aligned}
$$

\footnotetext{
${ }^{1}$ Rotation can be included by a suitable change of coordinates.
} 
and similarly for $\bar{L}_{0}, \bar{L}_{1}, \bar{L}_{-1}$ defined as (3.3) by substituting $u \rightarrow v$ and $v \rightarrow u$. Locally they form a basis of the Lie algebra $\mathrm{SL}(2, R)$,

$$
\left[L_{0}, L_{ \pm 1}\right]=\mp L_{ \pm 1}, \quad\left[L_{1}, L_{-1}\right]=2 L_{0} .
$$

Note that these vector fields cannot be integrated to generate global symmetries of the black hole background since they do not commute with the (hyperbolic) elements of $\mathrm{SL}(2, R)$ which provide the discrete identifications of points in $A d S_{3}$ required to produce the black hole (3.2). In particular, they do not preserve the boundary conditions when acting on functions defined on the black hole manifold. Nevertheless they turn out to be quite useful since, as we will now show, they generate the whole tower of quasi normal modes.

The vector fields $L_{k}$ and $\bar{L}_{k}$ satisfy the usual Killing equation $\nabla_{\mu} L_{\nu}+\nabla_{\nu} L_{\mu}=0$. In three dimensional locally AdS spacetime chacterised by the Riemann tensor $R_{\beta \mu \nu}^{\alpha}=$ $-\left(\delta_{\mu}^{\alpha} g_{\beta \nu}-\delta_{\nu}^{\alpha} g_{\beta \mu}\right)$ the Killing vectors satisfy in addition the following second order equations

$$
\begin{aligned}
& \nabla_{(\mu} \nabla_{\nu)} L_{\alpha}=g_{\mu \nu} L_{\alpha}-\frac{1}{2}\left(L_{\mu} g_{\nu \alpha}+L_{\nu} g_{\mu \alpha}\right) \\
& \nabla_{[\mu} \nabla_{\nu]} L_{\alpha}=\frac{1}{2}\left(L_{\mu} g_{\nu \alpha}-L_{\nu} g_{\mu \alpha}\right)
\end{aligned}
$$

Using these equations one shows that the Lie derivatives with respect to the Killing vectors $L_{k}$ are compatible with the zero trace, transversality condition (2.5) on $h_{\mu \nu}$, i.e. (2.5) implies

$$
g^{\mu \nu}\left(L_{k} h_{\mu \nu}\right)=0, \quad \nabla^{\mu}\left(L_{k} h_{\mu \nu}\right)=0, \quad k=0,-1,1 .
$$

Similarly the equation of motion (2.8) implies

$$
\left(\epsilon_{\mu}^{\alpha \beta} \nabla_{\alpha} h_{\beta \nu}+m\right) L_{k} h_{\mu \nu}=0
$$

\subsection{Scalar modes}

Before constructing the gravitational quasi-normal modes, as a warm-up we will reproduce the scalar quasi-normal modes [24, 25] in the BTZ background using the algebra of the Killing fields (3.4). The scalar field equation can be written in the form

$$
\left(-\nabla^{2}+m_{s}^{2}\right) \varphi=\left[2\left(L^{2}+\bar{L}^{2}\right)+m_{s}^{2}\right] \varphi=0
$$

where $L^{2}=\frac{1}{2}\left(L_{1} L_{-1}+L_{-1} L_{1}\right)-L_{0}^{2}$ is the Casimir operator of $\operatorname{SL}(2, R)$. In the process we will uncover a new structure present in the set of quasi-normal modes. In particular we will see that rather similar to the "highest weight" solutions in $A d S_{3}$ found in [26], the quasi-normal modes are descendents of a solution to a first order differential equation. The difference, however, is that only a half of the "highest weight" conditions should be imposed.

To see this we make the Ansatz

$$
\Phi=e^{-i p_{+} u-i p_{-} v} F(\rho)
$$

so that $L_{0} \Phi=i p_{+} \Phi$ and $\bar{L}_{0} \Phi=i p_{-} \Phi$, note that $\omega=p_{+}+p_{-}$and $k=p_{+}-p_{-}$are respectively the energy and the $\phi$-momentum. Following [26] one could impose the "highest 
weight" conditions $L_{1} \Phi=\bar{L}_{1} \Phi=0$ reflecting the fact that there are two $\operatorname{SL}(2, R)$ algebras locally present in the geometry. These conditions are perfectly suited to reproduce the so-called "normalizable modes" in anti-de Sitter space-time studied in [27] but they appear to be too strong in the black hole background because the descendents of such highest weight modes have imaginary $\phi$-momentum.

This problem can be avoided, however, by imposing a weaker condition which allows for real $\phi$-momentum, namely

$$
L_{1} \Phi=0
$$

This is exactly a half of the "highest weight" condition. Therefore, we call it a "chiral highest weight" condition. This condition then implies a first order equation for $F(\rho)$

$$
\left(2 i p_{+} \cosh (2 \rho)+2 i p_{-}+\sinh (2 \rho) \partial_{\rho}\right) F(\rho)=0 .
$$

If one were to impose in addition $\bar{L}_{1} \phi=0$, then it is clear from (3.4) that the the wave equation (3.9) reduces to an algebraic equation for $p_{ \pm}$. It is not obvious that this should still hold when imposing just one condition $L_{1} \Phi=0$. However, it turns out that this is indeed the case. The key observation is that

$$
\bar{L}_{1} \bar{L}_{-1} \Phi=\left(p_{+}^{2}-p_{-}^{2}+i\left(p_{+}+p_{-}\right)\right) \Phi .
$$

With this the scalar wave equation (3.9) reduces to the algebraic equation

$$
4 p_{+}^{2}+4 i p_{+}+m_{s}^{2}=0
$$

Notice that only $p_{+}$but not $p_{-}$appears in (3.14). Thus

$$
p_{+}=-i h_{L}\left(m_{s}\right), \quad h_{L}\left(m_{s}\right)=\frac{1+\sqrt{1+m_{s}^{2}}}{2} .
$$

If the BTZ black hole is interpreted as part of the gravitational dual to the $N=(4,4)$ superconformal field theory on the boundary, then $h\left(m_{s}\right)$ is the (left moving) conformal weight of the dual operator in the CFT on the boundary [25]. Reality of the $\phi$-momentum then requires $p_{+}-p_{-}=k$, where $k$ is real but otherwise unconstraint. If the direction along the coordinate $\phi$ is compact as it is for the BTZ case the momentum $k$ along this direction should be integer. The solution is then given by

$$
\begin{aligned}
\Phi & =e^{-2 h_{L}\left(m_{s}\right) \tau+i k(\tau-\phi)} F(\rho) \\
F(\rho) & =(\sinh (\rho))^{-2 h_{L}\left(m_{s}\right)}(\tanh (\rho))^{i k} .
\end{aligned}
$$

This solution is ingoing at the horizon, falls off in time as well as at infinity. The corresponding frequency

$$
\omega^{L}=-k-2 i h_{L}\left(m_{s}\right)
$$

is the lowest quasi-normal mode in the left-moving sector. Note that we define the leftand right-moving sectors from the boundary CFT point of view. 
The quasi-normal mode (3.16) determines the location of the pole, in the lower half plane, nearest to the real axis, of the retarded correlation function of a left-moving chiral operator, $V_{h_{L}}(\tau+\phi)$, in the boundary CFT with conformal weight $h_{L}(m)$ [25]. Note that the bulk quasi-normal mode corresponding to this pole is right moving.

For negative $m_{s}^{2}$ such that $-1 \leq m_{s}^{2}<0$ there exists second solution to the equation (3.4) corresponding to the conformal weight $h_{L}=\frac{1-\sqrt{1+m_{s}^{2}}}{2}$ that would produce a second set of quasi-normal modes. This is consistent with the observation made in [25].

Furthermore, provided $h_{L}\left(m_{s}\right) \neq 0$ then, acting by $L_{-1} \bar{L}_{-1}$ on (3.16) produces again a quasi-normal mode with the same asymptotic fall-off behaviour as (3.16) but with $\operatorname{Im} \omega \rightarrow$ $\operatorname{Im} \omega-2$. Thus, the condition $L_{1} \Phi=0$ together with an algebraic condition on $p_{+}$and $p_{-}$ leads to an infinite tower of quasi normal scalar modes

$$
\Phi^{(n)}=\left(L_{-1} \bar{L}_{-1}\right)^{n} \Phi
$$

that are descendents of the mode $\Phi$. In particular, the asymptotic fall-off behavior of $\Phi^{(n)}$ for large $\rho$ is uniquely determined by $h_{L}\left(m_{s}\right)$ and is independent of $n$.

The corresponding frequencies

$$
\omega_{n}^{L}=-k-2 i\left(h_{L}\left(m_{s}\right)+n\right), \quad n \in Z
$$

are exactly the quasi-normal frequences in this chiral sector found in 25, 24. For $h_{L}\left(m_{s}\right)=$ 0 solution (3.16) is not a quasi-normal mode since it does not satisfy the quasi-normal boundary condition.

If instead of (3.11) we impose $\bar{L}_{1} \Phi=0$ this leads to the first order equation for $F(\rho)$

$$
\left(2 i p_{-} \cosh (2 \rho)+2 i p_{+}+\sinh (2 \rho) \partial_{\rho}\right) F(\rho)=0
$$

Imposing the field equation the gives

$$
p_{-}=-i h_{R}\left(m_{s}\right)
$$

where $h_{R}\left(m_{s}\right)=h_{L}\left(m_{s}\right)$ for a scalar perturbation. The corresponding quasi-normal modes can be obtained form the previous one by the substitution $p_{+} \leftrightarrow p_{-}$which results in $\tau-\phi \rightarrow$ $\tau+\phi$ in (3.17) and thus reproduces the right-moving copy of the infinite tower (3.20). Again, repeated action by $L_{-1} \bar{L}_{-1}$ produces the whole tower of right moving quasi-normal modes,

$$
\omega_{n}^{R}=k-2 i\left(h_{R}\left(m_{s}\right)+n\right), \quad n \in Z
$$

in complete agreement with [25].

To summarize, we find that the complete set of scalar quasi normal modes in the BTZ background [25] is generated starting from the "chiral highest weight" conditions $L_{1} \Phi=0$ or $\bar{L}_{1} \Phi=0$. The remaining possibilities $\bar{L}_{-1} \Phi=0$ and $L_{-1} \Phi=0$ do not lead to quasi-normal modes since the solutions to this equation have outgoing flux at the horizon. 


\subsection{Tensor modes}

Let us now turn to the gravitational quasi-normal modes for topologically massive gravity. As explained in section 2, in the gauge (2.5) the equation for the massive graviton satisfies a first order equation of motion (2.8),

$$
\left(\epsilon_{\mu}^{\alpha \beta} \nabla_{\alpha} h_{\beta \nu}+m\right) h_{\mu \nu}=0
$$

We make the Ansatz [20] (in $u, v, \rho$ coordinates)

$$
h_{\mu \nu}=e^{-i p_{+} u-i p_{-} v} \psi_{\mu \nu}(\rho), \quad p_{+}+p_{-}=\omega, \quad p_{+}-p_{-}=k
$$

for the metric perturbation where

$$
\psi_{\mu \nu}=F(\rho)\left(\begin{array}{ccc}
1 & 0 & \frac{2}{\sinh (2 \rho)} \\
0 & 0 & 0 \\
\frac{2}{\sinh (2 \rho)} & 0 & \frac{4}{\sinh ^{2}(2 \rho)}
\end{array}\right) .
$$

The dominant component of $h_{\mu \nu}$ at infinity is $h_{u u}$. The transversality condition $\nabla_{\mu} h^{\mu}{ }_{\nu}=0$ then implies

$$
\left(2 i p_{+}+2 i p_{-} \cosh (2 \rho)+\sinh (2 \rho) \partial_{\rho}\right) F(\rho)=0 .
$$

A slightly tedious, but straight forward calculation shows that for our Ansatz the transversality condition is, in fact, equivalent to the "chiral highest weight" condition ${ }^{2}$

$$
\bar{L}_{1} h_{\mu \nu}=0
$$

In particular, equation (3.27) takes the form of the equation $\bar{L}_{1} F=0$ for scalar field $F$.

In addition we need to satisfy the first order equation of motion (2.8). Using $\epsilon^{\rho u v}=$ $\frac{1}{\sqrt{-g}}=\frac{4}{\sinh 2 \rho}$ we get from (2.8) for $\mu=\nu=\rho$ that

$$
p_{-}=-i h_{R}(m), \quad h_{R}(m)=-\frac{1}{2}-\frac{m}{2}
$$

where now $h_{R}(m)$ is the right-moving conformal weight (for negative $m$ ). For $m=-1$, the weight $h_{R}(m)$ vanishes. If condition (3.29) is satisfied then the remaining equations in (2.8) reduce to the transversality condition (2.5). It is clear that (2.8) implies (2.5) for any symmetric tensor. What we have just shown is that the converse holds true for the Ansatz (3.25) provided (3.29) is satisfied. The momentum in $\phi$-direction $k=p_{+}-p_{-}$ should be integer if $\phi$ is compact. We thus get

$$
h_{\mu \nu}=e^{-2 h_{R}(m) \tau-i k(\tau+\phi)} \psi_{\mu \nu}(\rho) .
$$

The solution (3.27) is then given by

$$
F(\rho)=(\sinh (\rho))^{-2 h_{R}(m)}(\tanh (\rho))^{-i k}
$$

\footnotetext{
${ }^{2}$ For a generic symmetric tensor $t_{\mu \nu}$ the transversality condition 3.27 can, of course, not be equivalent to $\bar{L}_{1} t_{\mu \nu}=0$ since the latter impose six constraint rather than three in (3.27).
} 
and thus the solution is completely determined. For $m<-1$ this solution is ingoing at the horizon and falls off at infinity as well as in time. It is thus a genuine quasi-normal mode. The Ansatz (3.25) for the metric perturbation together with the asymptotic behavior of (3.31) has the right asymptotic form in order to couple to the right moving component of a two-index tensor $T_{v v}$ in the boundary CFT since the $v \rho$ and the $\rho \rho$ components are subdominant at infinity. In what follows we will require this asymptotic structure for quasi-normal mode solutions.

In analogy with scalar modes we can act on the "chiral highest weight" quasi-normal mode with $L_{-1} \bar{L}_{-1}$. The effect of this will be to replace $\operatorname{Im} \omega \rightarrow \operatorname{Im} \omega-2$ in (3.30). As was discussed in section 3.1,

$$
h_{\mu \nu}^{(n)}=\left(L_{-1} \bar{L}_{-1}\right)^{n} h_{\mu \nu}
$$

is transverse and traceless. Furthermore, $L_{-1} \bar{L}_{-1}$ commutes with the equation of motion (2.8). Thus $h_{\mu \nu}^{(n)}$ is again a solution of the equation of motion with the same asymptotic fall-off behavior as $(3.30)$. Consequently, the complete tower of right-moving gravitational quasi-normal modes is generated in this way from the basic solution (3.30). The corresponding quasinormal frequencies are given by

$$
\omega_{n}^{R}=k-2 i\left(h_{R}(m)+n\right), \quad n \in Z
$$

For chiral gravity $m=-1$ the solution (3.30) has constant amplitude (in $\rho$ and $t$ ) and is thus not quasi-normal.

We can obtain a second solution by choosing

$$
\psi_{\mu \nu}=F(\rho)\left(\begin{array}{ccc}
0 & 0 & 0 \\
0 & 1 & \frac{2}{\sinh (2 \rho)} \\
0 & \frac{2}{\sinh (2 \rho)} & \frac{4}{\sinh ^{2}(2 \rho)}
\end{array}\right) .
$$

In this case we have

$$
\nabla_{\mu} h^{\mu}{ }_{\nu}=0 \Longrightarrow\left(2 i p_{-}+2 i p_{+} \cosh (2 \rho)+\sinh (2 \rho) \partial_{\rho}\right) F(\rho)=0
$$

which, in turn, is equivalent to $L_{1} h_{\mu \nu}=0$. Furthermore, the Ansatz (3.25) satisfies the first order equation of motion (2.8) provided

$$
p_{+}=-i h_{L}(m), \quad h_{L}(m)=\frac{m}{2}-\frac{1}{2} .
$$

Since $p_{+}-p_{-}=k$ we then get

$$
h_{\mu \nu}=e^{-2 h_{L}(m) \tau+i k(\tau-\phi)} \psi_{\mu \nu}(\rho)
$$

and

$$
F(\rho)=(\sinh (\rho))^{-2 h_{L}(m)}(\tanh (\rho))^{i k} .
$$

This is the basic quasi-normal mode for $m>1$. It couples to the left-moving components $T_{u u}$ of a 2-tensor. The higher quasi-normal modes are again obtained by acting with $L_{-1} \bar{L}_{-1}$. The corresponding frequencies are

$$
\omega_{n}^{L}=-k-2 i\left(h_{L}(m)+n\right), \quad n \in Z
$$


For chiral gravity $m=1$ the quasi-normal mode is absent. In analogy with the scalar modes the conditions $L_{-1} h_{\mu \nu}=0$ and $\bar{L}_{-1} h_{\mu \nu}=0$ lead to unphysical solutions with outgoing flux at the horizon.

\section{Conclusion}

In this paper we have found the quasi-normal mode spectrum for black holes in topologically massive gravity. We found that the quasi-normal modes satisfy a first order chiral highest weight equation. While the presence of a first order equation for gravitons can be understood from the form of the action, the fact that this equation agrees with a highest weight condition of the $\mathrm{SL}(2, R)$ algebra suggests that the highest weight condition should be understood as the implementation of the chirality condition on the gravitational excitations, ie. up to the exponential decay in time they only depend on $u$ or $v$. Since the gravitational excitations are chiral as consequence of the CS-term the equation of motion and the highest weight condition should coincide. That the same structure appears for the scalar QNM's is less obvious since they satisfy a second order differential equation.

We should also mention that for a given sign of $m$ there are only quasi-normal modes with definite chirality. Form the gravitational point of view it is clear that this has to be so because the CS-term in the action is odd under the exchange of $u$ and $v$. Thus given a solution $h_{\mu \nu}(\tau, v)$ the corresponding function $h_{\mu \nu}(\tau, u)$ is then necessarily a solution of the equation of motion of the action with the opposite sign of $m$. On the CFT side this leads to the interesting prediction that for $m<0$ the retarded Green function of the right moving primary $T_{u u}$, if it exists, has no poles in the lower half complex frequency plane which should then imply that the real-time Green function is quasi-periodic even at finite temperature. Similarly for $T_{v v}$ and $m>0$.

For chiral gravity $(m=1$ or $m=-1$ ) we did not find any quasi-normal modes. This may not be surprising since for these values of $m$ the tensor perturbations (3.24) satisfy the wave equation for massless gravitons which are known not to propagate. It should be noted that we only analysed solutions to the first order equation (2.8). There may be however solutions to the third order in derivative equation (2.7) that are not solutions to (2.8) or the massless graviton equation. In recent work [22] it was found that for $m^{2}=1$ equation (2.7) has a non-trivial solution which grows at the boundary. This solution, however, is not a quasi normal mode since it does not satisfy the QNM boundary conditions and grows in time. $^{3}$

\section{Acknowledgments}

I.S. an S.S. were supported in parts by the Transregio TRR 33 'The Dark Universe'. In addition I.S. acknowledges support from the Excellence Cluster 'Origin and Structure of the Universe' of the DFG as well as the DFG grant Ma 2322/3-1. S.S. thanks X. Bekaert for useful discussions and R. Metsaev for pointing out reference [17]. S.S. is grateful to the Theory Division at CERN for the hospitality extended to him while this work was completed.

\footnotetext{
${ }^{3}$ We thank D. Grumiller for raising this point.
} 


\section{References}

[1] A. Achucarro and P.K. Townsend, A Chern-Simons action for three-dimensional Anti-de Sitter supergravity theories, Phys. Lett. B 180 (1986) 89.

[2] E. Witten, $(2+1)$-dimensional gravity as an exactly soluble system, Nucl. Phys. B 311 (1988) 46.

[3] M. Bañados, Global charges in Chern-Simons field theory and the $(2+1)$ black hole, Phys. Rev. D 52 (1996) 5816 hep-th/9405171.

[4] O. Coussaert, M. Henneaux and P. van Driel, The asymptotic dynamics of three-dimensional Einstein gravity with a negative cosmological constant, Class. and Quant. Grav. 12 (1995) 2961 gr-qc/9506019.

[5] M. Bañados, C. Teitelboim and J. Zanelli, The black hole in three-dimensional space-time, Phys. Rev. Lett. 69 (1992) 1849 hep-th/9204099.

[6] S. Hyun, Y.-S. Song and J.H. Yee, Hawking radiation of Dirac fields in the $(2+1)$-dimensional black hole space-time, Phys. Rev. D 51 (1995) 1787 [hep-th/9409047].

[7] D. Birmingham, I. Sachs and S. Sen, Three-dimensional black holes and string theory, Phys. Lett. B 413 (1997) 281 hep-th/9707188.

[8] R. Emparan and I. Sachs, Quantization of $A d S_{3}$ black holes in external fields, Phys. Rev. Lett. 81 (1998) 2408 hep-th/9806122.

[9] S. Carlip, What we don't know about BTZ black hole entropy, Class. and Quant. Grav. 15 (1998) 3609 hep-th/9806026.

[10] A. Strominger and C. Vafa, Microscopic origin of the Bekenstein-Hawking entropy, Phys. Lett. B 379 (1996) 99 hep-th/9601029.

[11] J.M. Maldacena, Eternal black holes in Anti-de-Sitter, JHEP 04 (2003) 021 hep-th/0106112.

[12] D. Birmingham, I. Sachs and S.N. Solodukhin, Relaxation in conformal field theory, Hawking-Page transition and quasinormal/normal modes, Phys. Rev. D 67 (2003) 104026 hep-th/0212308.

[13] J. Manschot and G.W. Moore, A modern Farey tail, arXiv:0712.0573.

[14] O. Lunin and S.D. Mathur, AdS/CFT duality and the black hole information paradox, Nucl. Phys. B 623 (2002) 342 hep-th/0109154].

[15] E. Witten, Three-dimensional gravity revisited, arXiv:0706.3359.

[16] S. Deser, R. Jackiw and S. Templeton, Three-dimensional massive gauge theories, Phys. Rev. Lett. 48 (1982) 975; Topologically massive gauge theories, Ann. Phys. (NY) 140 (1982) 372 [Erratum ibid. 185 (1988) 406] Ann. Phys. (NY) 281 (2000) 409.

[17] I.V. Tyutin and M.A. Vasiliev, Lagrangian formulation of irreducible massive fields of arbitrary spin in $(2+1)$ dimensions, Teor. Mat. Fiz. 113 (1997) 1244 hep-th/9704132].

[18] P. Kraus and F. Larsen, Holographic gravitational anomalies, JHEP 01 (2006) 022 hep-th/0508218.

[19] S.N. Solodukhin, Holography with gravitational Chern-Simons, Phys. Rev. D 74 (2006) 024015 hep-th/0509148. 
[20] W. Li, W. Song and A. Strominger, Chiral gravity in three dimensions, JHEP 04 (2008) 082 arXiv:0801.4566.

[21] S. Carlip, S. Deser, A. Waldron and D.K. Wise, Cosmological topologically massive gravitons and photons, arXiv:0803.3998.

[22] D. Grumiller and N. Johansson, Instability in cosmological topologically massive gravity at the chiral point, arXiv:0805.2610.

[23] M.-I. Park, Constraint dynamics and gravitons in three dimensions, arXiv:0805.4328.

[24] D. Birmingham, Choptuik scaling and quasinormal modes in the AdS/CFT correspondence, Phys. Rev. D 64 (2001) 064024 hep-th/0101194.

[25] D. Birmingham, I. Sachs and S.N. Solodukhin, Conformal field theory interpretation of black hole quasi-normal modes, Phys. Rev. Lett. 88 (2002) 151301 hep-th/0112055.

[26] J.M. Maldacena and A. Strominger, $A d S_{3}$ black holes and a stringy exclusion principle, JHEP 12 (1998) 005 hep-th/9804085.

[27] V. Balasubramanian, P. Kraus and A.E. Lawrence, Bulk vs. boundary dynamics in Anti-de Sitter spacetime, Phys. Rev. D 59 (1999) 046003 hep-th/9805171. 